\title{
Responsabilidad Social Empresarial: un instrumento para medir la implementación en las empresas del índice de sustentabilidad de la Bolsa Mexicana de Valores*
}

Corporate Social Responsibility: An instrument to measure the implementation in companies of the sustainability index of the Mexican Stock Exchange

Yara Landázuri Aguilera ${ }^{\text {a }}$

Instituto Tecnológico de Sonora, México

ylandazuri@itson.edu.mx

ORCID: http://orcid.org/0000-0002-7784-2762

DOI: https://doi.org/10.11144/Javeriana.cc19-48.rsei Redalyc: http://www.redalyc.org/articulo.oa?

Adriana Verónica Hinojosa Cruz

Universidad Autónoma de Nuevo León, México

ORCID: http://orcid.org/0000-0002-2225-4488 id $=151557795005$

Norma Aguilar Morales

Universidad Juárez Autónoma de Tabasco, México

ORCID: http://orcid.org/0000-0002-7292-3029

Fecha de recepción: 30 Enero 2018

Fecha de aprobación: 25 Junio 2018

Fecha de publicación: 20 Diciembre 2018

\section{Resumen:}

Este artículo presenta el diseño y la validación de un instrumento de medición de la responsabilidad social empresarial a través de las dimensiones económica, social y medioambiental, utilizando técnica de mínimos cuadrados parciales (PLS) y el software utilizado para tal fin ha sido Smart-PLS, versión 3. La investigación se realizó con la información de una muestra de empresas que cotizan en la Bolsa Mexicana de Valores y que pertenecen al índice de sustentabilidad. Los resultados confirman un instrumento estadísticamente válido para medir la responsabilidad social a través de las tres dimensiones propuestas con oportunidades de mejora para dimensión económica y de esta manera continuar con la investigación sobre el tema de responsabilidad social en México.

Palabras clave: dimensiones, instrumento de medición, responsabilidad social empresarial, validez, Bolsa Mexicana de Valores.

\section{Abstract:}

This article presents the design and validation of a measuring instrument for corporate social responsibility through the economic, social and environmental dimensions, with the technique of Partial Least Squares (PLS) and Smart-PLS soft ware, version 3. The research was carried out with information from a sample of companies that are listed on the Mexican Stock Exchange and that belong to the sustainability index. The results confirm a statistically valid instrument to measure social responsibility through the three proposed dimensions with opportunities for improvement for economic dimension and thus continue with research on the issue of social responsibility in Mexico. Keywords: Dimensions, measuring instrument, corporate social responsibility, validity, Mexican Stock Exchange.

\section{Introducción}

La Responsabilidad Social Empresarial (RSE) ha despertado el interés no sólo de las empresas, sino también de analistas, críticos, consultores, gobierno y la sociedad civil. Actualmente, la relevancia sobre el tema de la Responsabilidad Social no se pone en duda, sin embargo, en México aún existen vacíos sobre la implementación y comunicación de las acciones socialmente responsables que llevan a cabo las grandes empresas, el destino de los montos económicos y el impacto que puede generar en ellas.

Los estudios existentes han evolucionado desde investigaciones básicas sobre las definiciones de la RSE y su evolución, hasta tratar de medir el impacto en las mismas empresas que realizan actividades socialmente

Notas de autor

a Autora de correspondencia. Correo electrónico: ylandazuri@itson.edu.mx 
responsables. En la literatura se han logrado identificar estudios aplicados que explican la relación que existe entre las medidas de RSE adoptadas por las empresas y sus resultados empresariales de largo plazo (Tsoutsoura, 2004; De la Cuesta, 2004; Porter \& Kramer, 2006; Hull \& Rothenberg, 2008; Baumgartner \& Ebner, 2010; Chow \& Chen, 2011; Vicente, Tamayo \& Izaguirre, 2012; Larrán, Herrera \& Martínez, 2013; Dumitrescu \& Simionescu, 2015; Saeidi et ál., 2015; Xie et ál., 2017).

Llama la atención que uno de los principales problemas que aparecen en la revisión de la literatura sobre el tema es la medición, los investigadores en este campo consideran que la RSE es un concepto multidimensional, logrando presentar sus propuestas sobre los efectos de la RSE en el desempeño en las empresas, pero no han podido determinar la participación que tiene en cada dimensión.

El propósito central de esta investigación consiste en el diseño y la validación de un instrumento de elaboración propia -que considera aportes de la literatura- por los siguientes motivos: (i) el instrumento ha sido elaborado para desarrollar un trabajo de investigación sobre las acciones de RSE que llevan a cabo las empresas mexicanas, (ii) porque en la literatura no existe un instrumento que mida la RSE en las empresas mexicanas, los estudios empíricos que se han realizado han sido con base en índices (indicadores sustentables, índices asociados al mercado o financieros) y en su mayoría de países extranjeros, (iii) la investigación se realiza a través de las tres dimensiones evaluadas por el Global Reporting Initiative (económica, social y medioambiental).

$\mathrm{El}$ instrumento considera ítems que pretenden medir cuatro aspectos relevantes de la RSE, como son: (i) incorporación de la RSE en las funciones empresariales y su vinculación con los grupos de interés, (ii) su contribución para mejorar la calidad de vida de los empleados y las comunidades en las que opera, (iii) la regulación de sus actividades a través de normas internacionales y difusión, (iv) las prácticas interna y externa sobre los impactos medibles de las acciones de RSE destinadas a preservar el entorno.

\section{Revisión de la literatura}

Hemos dividido la revisión de la literatura en dos secciones, en la primera presentamos los estudios relacionados con la evolución de la responsabilidad social empresarial; y en la segunda, la medición a través de las tres dimensiones de responsabilidad social que se ha identificado en la literatura.

\section{Evolución de los estudios sobre RSE}

La responsabilidad social empresarial (RSE) aparece de forma explícita en la segunda mitad del siglo XX. Sin embargo, se puede afirmar que en términos éticos y morales esta filosofía ha estado presente desde los inicios de la historia de la humanidad a través de normativas para garantizar correctas prácticas comerciales (Canessa \& García, 2005). La RSE se ha fortalecido con el tiempo, las primeras actividades estaban relacionadas con el apoyo mutuo, reconocidas como acciones altruistas o filantrópicas. Sin embrago es a partir de la publicación de Bowen (1953), cuando la RSE toma forma y es percibida como un medio para mejorar la gestión organizacional al tiempo que estas intentan contrarrestar los problemas económicos y alcanzar sus objetivos.

De esta manera se detonó el interés por parte de las empresas para tratar de mitigar algunos de los problemas sociales a través de su participación activa, identificado que sus acciones generan impacto positivo en la sociedad. Estudios como los de Orlitzky, Schmidt y Rynes (2003), Marín y Rubio (2008), Sánchez y Hernández (2014), Saeidi et ál. (2015), Xie et ál. (2017), Rodrigues y Mendes (2018), manifiestan que las empresas socialmente responsables obtienen mayor beneficio de largo plazo a consecuencia de una mejor aceptación por parte de sus stakeholders. Si bien Kurapatskie y Darnall (2013) plantean un vínculo estrecho 
entre la RSE y el rendimiento de empresas, persiste la controversia con respecto al impacto de la RSE sobre el desempeño financiero.

El estudio de Ramos, Manzanares y Gómez (2014) muestra que los estudios empíricos sobre la RSE han evolucionado, identificando dicha evolución desde el interés y la manera de poner en práctica las iniciativas de responsabilidad social, hasta los estudios aplicados que intentan probar la relación que existe entre las medidas de RSE adoptadas por las empresas y sus resultados empresariales a largo plazo como De la Cuesta (2004), Porter y Kramer (2006), Hull y Rothenberg (2008), Baumgartner y Ebner (2010) y Chow y Chen (2011), Vicente, Tamayo e Izaguirre (2012), Larrán, Herrera y Martínez (2013), Trumpp y Guenther (2017), Rhou, Singal y Koh (2016), García, Ruiz y López (2017).

En la última década las empresas han mostrado un interés creciente por la implementación de la RSE, como potente herramienta de gestión desde un punto de vista estratégico (Porter \& Kramer, 2006; Surroca, Tribó \& Waddock, 2010; Donate \& Guadamillas, 2011; Rhou et ál., 2016). Además los estudios han involucrado nuevas variables con el fin de obtener una mejor explicación sobre los efectos de la responsabilidad social, por ejemplo Saeidi et al. (2015) examinan el efecto de mediación de la ventaja competitiva sostenible, la reputación y la satisfacción del cliente en la relación con la RSE y el rendimiento financiero, para hallar un efecto indirecto de la RSE en el aumento del rendimiento de la empresa a través de una ventaja competitiva, reputación y satisfacción del cliente mejoradas.

Uno de los principales avances en el tema de Responsabilidad Social Empresarial en México ha sido el Centro Mexicano para la Filantropía (Cemefi), creado en 1997, con la intención de generar un programa en Responsabilidad Social Empresarial para certificar a las empresas que operan en México. Con base en esta iniciativa, en el año 2000 el Cemefi abrió la primera convocatoria para reconocer las mejores prácticas de RSE, y en 2001 se otorgó por primera vez el distintivo Empresa Socialmente Responsable a 17 empresas.

En la literatura existen diversos marcos referenciales para explicar el concepto de responsabilidad social empresarial y sus dimensiones. El modelo propuesto por Panwar, Rinne, Hansen y Juslin (2006) resulta ser el más apropiado para esta investigación, debido a la identificación de estudios empíricos recientes que clasifican la responsabilidad social empresarial en las mismas tres dimensiones (económica, social y medioambiental) que hacen los autores anteriormente citados, destacando a Vázquez y Hernández (2013) y Ramos, Manzanares Gómez (2014) y Rodrigues y Mendes (2018).

La tabla 1 presenta la clasificación que se aborda en esta investigación, así como la definición de cada variable evaluada para el estudio y análisis de la responsabilidad social empresarial. 
TABLA 1

Definición de las dimensiones de la RSE

\begin{tabular}{|c|c|c|}
\hline Dimensión & Variable / Definición & Referencias \\
\hline $\begin{array}{l}\text { Dimensión } \\
\text { Económica }\end{array}$ & $\begin{array}{l}\text { Interna. Es la generación y distribución del valor agregado } \\
\text { entre colaboradores (empleados), clientes, proveedores y } \\
\text { accionistas, considerando no sólo las condiciones de } \\
\text { mercado, sino tambiénla equidady la justicia. Se espera que } \\
\text { la empresa genere utilida des y mantenga finanzas sanas a } \\
\text { través de prácticas éticas y se mantenga activa } \\
\text { indefinidamente. } \\
\text { Externa. Implica la generación y distribución de bienes y } \\
\text { servicios para la comunidad, la aportación a la causa pública } \\
\text { vía contribución impositiva y la influencia en la implantación } \\
\text { de la política económica de su región. }\end{array}$ & $\begin{array}{l}\text { Spiller }(2000) \text {, } \\
\text { Cajiga (2009), } \\
\text { Mishra y Suar (2010) }\end{array}$ \\
\hline Dimensión Social & $\begin{array}{l}\text { Interna. Es la responsabilidad compartida y subsidiaria de } \\
\text { inversionistas, directivos, colaboradores (empleados), } \\
\text { clientes y proveedores, para el cuidado y fomento de la } \\
\text { calidad de vida en el trabajo y el desarrollo integral y pleno } \\
\text { de todos ellos. } \\
\text { Externa. Es la realización de acciones y aportaciones propias } \\
\text { y colectivas para contribuir con tiempo y recursos a la } \\
\text { generación de condiciones que permitany favorezcan la } \\
\text { expansión del espinitu empresarial y el pleno desarrollo de las } \\
\text { comunidades donde operan. }\end{array}$ & $\begin{array}{l}\text { Cajiga (2009), } \\
\text { Mishra y Suar (2010), } \\
\text { Larrán, Herrera y } \\
\text { Martínez (2013) }\end{array}$ \\
\hline $\begin{array}{l}\text { Dimensión } \\
\text { Medioambiental }\end{array}$ & $\begin{array}{l}\text { Interna. Implica la mejora del desempeño ambiental de la } \\
\text { empresa con base en un enfoque de eco-eficiencia y } \\
\text { prevención que maximice el uso de insumos y recursos } \\
\text { naturales. } \\
\text { Externa. La promoción de la protección y restauración del } \\
\text { patrimonio ambiental, la promoción de los principios y } \\
\text { valores correspondientes a lo largo de la cadena de valor en } \\
\text { que participa la empresa y, en caso necesario, remediar los } \\
\text { daños ambientales que ocasionen sus procesos productivos. }\end{array}$ & $\begin{array}{l}\text { Cajiga (2009), } \\
\text { Mishra y Suar (2010), } \\
\text { Larrán, Herrera y } \\
\text { Martínez (2013) }\end{array}$ \\
\hline
\end{tabular}

Fuente: elaboración propia.

La responsabilidad social empresarial (RSE) puede ser estudiada a través de diferentes perspectivas, las cuales varían dependiendo del alcance de cada investigación que se pretende realizar. Para esta investigación la RSE ha sido considerada como una variable de segundo orden, dado que teóricamente está formada por otras tres dimensiones suficientemente diferenciadas: las dimensiones económica, social y medioambiental.

\section{Medición de la RSE}

La forma en que lleva a cabo la medición de las acciones de responsabilidad social en los meta-análisis identificados en la literatura, han sido en su mayoría a través de principios de sustentabilidad basados en indicadores de organismos internacionales dedicados a promover estas prácticas, indicadores bursátiles especializados en sustentabilidad, informes sociales por parte de las empresas u organismos involucrados en el reconocimiento de estas, rankings de reputación, publicaciones en revistas o boletines en los que se reconoce la labor de estas empresas, entre otros. Cuando los indicadores disponibles no resultan útiles debido a que el ámbito de la investigación es específico de alguna región o sector para el que no existen instrumentos válidos para el levantamiento de datos o bien las medidas existentes no satisfacen las necesidades de investigación, 
algunos autores deciden elaborar medidas propias a partir de literatura teórica y empírica en RSE (Ramos, Manzanares \& Gómez, 2014).

De acuerdo con el posicionamiento de los autores, investigaciones como las de Bansal (2005), Jackson y Apostolakou (2010), Chow y Chen (2011), Vázquez y Hernández (2013) y Ramos et ál. (2014), se han adentrado en el campo de la medición con el fin de diseñar sus propios instrumentos de medida que permitan cuantificar y evaluar las acciones socialmente responsables en las empresas a través de las dimensiones económica, social y medioambiental.

Para esta investigación se ha realizado una selección de ítems generando con esto un hibrido entre los cuestionarios identificados en la literatura para el diseño de nuestro propio instrumento, el cual es considerado en esta investigación como un aporte a la literatura de la RSE. A continuación se indican los referentes considerados de la literatura para el diseño de los ítems que componen el instrumento de medición de la RSE que se validará a través de la herramienta estadística SPSS, Versión 21, bajo el análisis factorial y alfa de Cronbach, seleccionando los ítems que resulten con mejores cargas y resultados estadísticos aceptables por la literatura para el estudio y alcanzando con esto el principal objetivo que da origen a esta investigación.

TABLA 2

Ítems de responsabilidad social empresarial

\begin{tabular}{|c|c|c|}
\hline Variable & Unidad de medición & Autores \\
\hline $\begin{array}{l}\text { Dimensión } \\
\text { Económica }\end{array}$ & $\begin{array}{l}9 \text { items que miden intema y } \\
\text { extemamente la dimensión } \\
\text { económica de la empresa, con una } \\
\text { escala tipo Likert de } 6 \text { puntos. }\end{array}$ & $\begin{array}{l}\text { De la Cuesta (2004), Porter y Kramer (2006), } \\
\text { Alvara do y Schlesinger (2008), Alvara do } \\
\text { (2008), Baumgartnery Ebner (2010), Chow y } \\
\text { Chen(2011), González, Donate y Guadamillas } \\
\text { (2014). }\end{array}$ \\
\hline $\begin{array}{l}\text { Dimensión } \\
\text { Social }\end{array}$ & $\begin{array}{l}9 \text { items que miden intema y } \\
\text { extemamente la dimensión social de } \\
\text { la empresa, con una escala tipo } \\
\text { Likert de } 6 \text { puntos }\end{array}$ & $\begin{array}{l}\text { Bansal(2005), García de los Salmones, } \\
\text { Rodríguez del Bosque y San Martín(2007), } \\
\text { Alvarado y Schlesinger (2008), Alvara do } \\
\text { (2008), Lindgreen, Antioco, Hamess y Van } \\
\text { der Sloot (2009), Baumgartnery Ebner } \\
\text { (2010), Chow y Chen (2011), Ramos, } \\
\text { Manzanares y Gómez (2014). }\end{array}$ \\
\hline $\begin{array}{l}\text { Dimensión } \\
\text { Medioambiental }\end{array}$ & $\begin{array}{l}9 \text { items que miden intema y } \\
\text { extemamente la dimensión } \\
\text { medioambiental de la empresa, con } \\
\text { una escala tipo Likert de } 6 \text { puntos }\end{array}$ & $\begin{array}{l}\text { Bansal(2005), Jones y Comfort (2005), } \\
\text { Alvarado (2008), Erol Caka, Erel y Sari } \\
\text { (2009), Chow y Chen (2011), Ramos, } \\
\text { Manzanares y Gómez (2014). }\end{array}$ \\
\hline
\end{tabular}

Fuente: elaboración propia.

Con los ítems diseñados se intenta medir la importancia que la empresa otorga a las dimensiones de RSE y el grado de intensidad con que las aplica en la práctica. Se espera que cuanto mayor sea la importancia concedida a las actividades de RSE en cada una de sus dimensiones y mayor sea su implementación en la empresa, mejores resultados económicos, sociales y medioambientales derivados de la RSE se obtendrán para la empresa. Así, el instrumento propuesto para medir la RSE a través de sus tres dimensiones consta de los siguientes ítems. 
TABLA 3

Listado de ítems (propuesta)

\begin{tabular}{|c|c|}
\hline Variable & Ítems \\
\hline Dimensión económica & $\begin{array}{l}\text { Ec1 Rentabilidad de largo plazo } \\
\text { Ec2 Proyectos para mejorarla calidad } \\
\text { Ec3 Costos de insumos } \\
\text { Ec4 Costos administrativos } \\
\text { Ec5 Otros ingresos } \\
\text { Ec6 Programa de mercadeo } \\
\text { Ec7 Atención a clientes } \\
\text { Ec8 Apoyo a proveedores locales } \\
\text { Ec9 Proveedores certificados }\end{array}$ \\
\hline Dimensión social & $\begin{array}{l}\text { Soc10 Informes de sustentabilidad } \\
\text { Soc11 Patrocinios } \\
\text { Soc12 Apoyo a iniciativas locales } \\
\text { Soc13 Saludy seguridad en la comunidad } \\
\text { Soc14 Calidad de vida en la comunidad } \\
\text { Soc15 Saludy seguridad a empleados } \\
\text { Soc16 Igualdad salarial } \\
\text { Soc17 Lealtad de empleados } \\
\text { Soc18 Contratacionigualitaria }\end{array}$ \\
\hline Dimensión medioambiental & $\begin{array}{l}\text { Ma19 Impacto ambiental } \\
\text { Ma20 Informes medioambientales } \\
\text { Ma21 Reducción del impacto ambiental } \\
\text { Ma22 Accidentes ambientales } \\
\text { Ma23 Aprovechamiento de residuos } \\
\text { Ma24 Alianzas ambientales } \\
\text { Ma25 Politicas medioambientales } \\
\text { Ma26 Rescate y conservación del medioambiente } \\
\text { Ma27 Protección del medio ambiente }\end{array}$ \\
\hline
\end{tabular}

Fuente: elaboración propia.

Las investigaciones coinciden en la importancia de tener un equilibrio en las tres dimensiones económica, social y medioambiental de la RSE, considerando que estas serán estadísticamente analizadas de manera individual pero como parte del mismo constructo.

\section{Metodología de investigación}

De acuerdo con la estructura planteada para el desarrollo de esta investigación y con el propósito de alcanzar el objetivo principal, el trabajo se centra la validación de un instrumento que mida el grado de RSE adoptado por las empresas desde un punto de vista económico, social y medioambiental. Para ello, se han empleado dos técnicas: la primera es a través de la herramienta estadística SPSS, Versión 21. Con esta herramienta se estarán sometiendo los ítems diseñados a un análisis factorial por el método de rotación Varimax con Kaiser para la selección de los ítems y el análisis del alfa de Cronbach, también para seleccionar los ítems con mejores cargas factoriales. La segunda técnica es la de mínimos cuadrados parciales (PLS), por ser especialmente útil cuando se trata de analizar constructos formativos. Para esto se utilizó el software Smart-PLS, versión 3. Con esta segunda técnica se pretende comparar la selección de los ítems que se quedan en el instrumento para continuar con la investigación y verificar si los ítems eliminados coinciden en las dos técnicas. 


\section{Población}

El presente estudio está dirigido a las Empresas Socialmente Responsables en México, (i) que emitan acciones en la Bolsa Mexicana de Valores (BMV), (ii) que pertenecen al índice de sustentabilidad y (iii) que hagan públicos sus informes de sustentabilidad a través de sus páginas oficiales. Las 30 empresas pertenecientes a la muestra del Índice S\&P/BMV IPC Sustentable conforman la población de este estudio, ver tabla 4.

TABLA 4

Población del estudio: Empresas del índice sustentable

\begin{tabular}{ll}
\hline AC & HCITY \\
ALFA & HERDEZ \\
ALSEA & IENOVA \\
AMX & KIMBER \\
ASUR & KOF \\
AXTEL & LAB \\
BIMBO & LALA \\
BOLSA & MEXCHEM \\
BSMX & OMA \\
CEMEX & PE\&OLES \\
ELEKTRA & RASSINI \\
FEMSA & TLEVISA \\
GENTERA & VESTA \\
GFNORTE & VOLAR \\
GMEXICO & WALMEX \\
\hline
\end{tabular}

Fuente: elaboración propia.

El levantamiento de datos se realizó a través de un cuestionario en línea enviado a gerentes y responsables de la implementación de la RSE en las empresas; después de realizar contacto y seguimiento telefónico, se obtuvieron 19 cuestionarios válidos, que representan el 63\% de tasa de respuesta, es un porcentaje aceptable de acuerdo con la literatura, la cual considera válida una tasa de respuesta comprendida entre el 10 y el 20 por ciento en trabajos empíricos con levantamiento de datos a través de cuestionarios (Chow \& Chen, 2011).

\section{Método de análisis}

Para esta investigación se establece la RSE como un constructo de segundo orden de tipo reflectivo-formativo. En este sentido, se determina que el primer orden de las tres dimensiones es reflectivo, es decir; si varía cualquiera de los ítems de una dimensión en concreto, el resto de ítems que miden esta dimensión variarán en el mismo sentido. En el siguiente paso, se determina que el segundo orden es formativo, ya que si varía una de las dimensiones del constructo va a variar el significado global del mismo.

Para ello, se han empleado dos técnicas la primera de ellas es a través del SPSS, el cual es uno de los programas estadísticos más reconocidos por los investigadores para la comprobación de hipótesis en el campo de las ciencias sociales. Esta herramienta será utilizada con la finalidad de realizar el análisis factorial y el alfa de Cronbach para determinar un instrumento válido y fiable. La segunda herramienta estadística del SmartPLS, versión 3, donde se determina la relación entre los indicadores y el constructo latente y además se realiza 
la estimación del modelo estructural, donde se evalúan las relaciones entre los constructos, a través de los coeficientes y su nivel de significancia.

Es preciso señalar que, dado que el principal objetivo de este trabajo es validar el instrumento propuesto para medir la RSC y no se contemplan más variables ni se establece ninguna hipótesis, solamente se analiza y propone el modelo de medida, el cual permite evaluar la validez de dicho instrumento.

\section{Resultados estadísticos}

Los resultados se presentan en dos partes: la primera muestra los resultados obtenidos bajo el análisis de los resultados por el SPSS, versión 21, de cual se desprenden primeramente el analisis factorial y seguido el alfa de Cronbach. La segunda parte es a través del Smart-Pls, versión 3, en la que se analizan la validez, fiabilidad y colinealidad, posteriormente se realiza una propuesta de modelación de las tres dimensiones de la RSE, donde cada dimensión es una variable independiente.

\section{Análisis factorial}

Se realizó el análisis factorial a través del SPSS, introduciendo los 27 ítems que miden las tres dimensiones, de los cuales el resultado fue continuar el análisis de los datos con 15 ítems, debido a que alguno de estos eran reconocidos en más de un componente; una vez eliminados se obtuvo que la varianza explica el $73 \%$ de los datos, por lo tanto el porcentaje es aceptable de acuerdo con la teoría, la cual considera que existe validez convergente cuando la varianza alcanza valores superiores a 0,50 (Fornell \& Larcker, 1981; Chin, 1998); por lo tanto es posible trabajar con este número de ítems.

TABLA 5

Varianza explicada

\begin{tabular}{|c|c|c|c|c|c|}
\hline \multicolumn{3}{|c|}{$\begin{array}{c}\text { Sumas de las saturaciones al cuadrado de la } \\
\text { extracción }\end{array}$} & \multicolumn{3}{|c|}{$\begin{array}{c}\text { Suma de las saturaciones al cuadrado de la } \\
\text { rotación }\end{array}$} \\
\hline Total & $\begin{array}{c}\% \text { de la } \\
\text { varianza }\end{array}$ & $\%$ acumulado & Total & $\begin{array}{c}\% \text { de la } \\
\text { varianza }\end{array}$ & $\%$ acumulado \\
\hline 6,768 & 45,123 & 45,123 & 4,564 & 30,428 & 30,428 \\
\hline 2,745 & 18,298 & 63,421 & 3,273 & 21,821 & 52,248 \\
\hline 1,499 & 9,994 & 73,415 & 3,175 & 21,167 & 73,415 \\
\hline
\end{tabular}

Fuente: elaboración propia.

Una vez analizada la varianza se presenta la matriz de los componentes rotados en la cual se identifican los ítems que se quedan dentro del instrumento propuesto, los ítems eliminados fueron: E2, E3, E6, E7, E8, S10, S16, S17, S19, M19, M20 y M24, en esta primera prueba se identificaron 12 ítems que cargan en más de un componente, por lo que vale la pena considerar si se quedan o se eliminan del instrumento, quedando como propuesta a través del análisis factorial los ítems que se muestran en la tabla de la matriz de componentes rotados. 
TABLA 6

Matriz de componentes rotados

\begin{tabular}{lccc}
\hline & \multicolumn{3}{c}{ Componente } \\
\cline { 2 - 4 } & 1 & 2 & 3 \\
\hline E1 & 0,337 & 0,670 & 0,314 \\
E4 & 0,263 & 0,795 & 0,291 \\
E5 & 0,313 & 0,824 & 0,006 \\
E9 & 0,099 & 0,883 & 0,105 \\
\hline S11 & 0,234 & 0,365 & 0,749 \\
S12 & 0,407 & 0,267 & 0,678 \\
S13 & 0,050 & 0,054 & 0,910 \\
S14 & $-0,192$ & $-0,047$ & 0,804 \\
S15 & 0,109 & 0,473 & 0,645 \\
\hline M21 & 0,784 & 0,327 & 0,035 \\
M22 & 0,909 & 0,139 & 0,182 \\
M23 & 0,640 & 0,146 & 0,113 \\
M25 & 0,845 & 0,104 & $-0,072$ \\
M26 & 0,836 & 0,154 & 0,038 \\
M27 & 0,860 & 0,342 & 0,126 \\
\hline
\end{tabular}

Fuente: elaboración propia.

\section{Alfa de Cronbach}

Para esta investigación la determinación de la fiabilidad se realizó a través del alfa de Cronbach, apoyados en la herramienta estadística SPSS, versión 21, el objetivo es verificar que la mayor parte de los ítems diseñados para cada variable permitan alcanzar alfas con valores aceptables, es decir, superiores a 0,70 , considerado como mínimo aceptable para investigaciones científicas, de acuerdo con Sánchez y Gómez (1998) y Cervantes (2005).

Es importante señalar que para realizar la prueba de validez y lograr obtener los mejores valores de alfas de Cronbach fue necesario eliminar algunos ítems en las variables; procedimiento que se conoce como validez de contenido. A continuación se presentan los resultados del alfa de Cronbach con los ítems eliminados de acuerdo con los resultados de validez. 
TABLA 7

Alfa de Cronbach

\begin{tabular}{llcc}
\hline \multicolumn{1}{c}{ Variable } & Ítems eliminado & Ítems finales & Alfa de Cronbach \\
\hline & & E1 & 0,860 \\
Dimensión & \multirow{2}{*}{ E2, E6, E7 } & E3 & 0,881 \\
Económica & & E4 & 0,855 \\
& & E5 & 0,869 \\
& & E9 & 0,874 \\
& & S11 & 0,859 \\
\hline \multirow{2}{*}{ Dimensión Social } & \multirow{2}{*}{ Soc10, Soc16 } & S12 & 0,820 \\
& & S13 & 0,815 \\
& & S14 & 0,841 \\
& & S15 & 0,816 \\
Dimensión & & S17 & 0,858 \\
Medioambiental & Ma19,Ma20,Ma23 & M22 & 0,870 \\
& & M24 & 0,862 \\
& & M25 & 0,891 \\
& & M26 & 0,882 \\
\end{tabular}

Fuente: elaboración propia.

Los resultados de las alfas de Cronbach para las variables son aceptables de acuerdo con la teoría. Valores superiores a 0,70 y menores a 0,90 , son considerados como valores aceptables para investigaciones científicas en el campo de las ciencias sociales, de acuerdo con Sánchez y Gómez (1998) y Cervantes (2005), los ítems que conforman el cuestionario no están correlacionados y son independientes.

De los resultados analizados a través del SPSS se identifica que tanto para el análisis factorial como para el análisis de fiabilidad a través del alfa de Cronbach la eliminación de los ítems E2, E6, E7 se mantienen igual en la dimensión económica, para la dimensión social los ítems que se propone eliminar y que coinciden en los dos análisis son S10, S16 y S18, y para la dimensión medioambiental es M20.

El Smart-PLS es un modelo de análisis que utiliza el proceso de estimación en dos pasos (Gefen y Straub, 2005). El primero de ellos estima el modelo de medida y se determina la relación entre los ítems o indicadores y la variable latente. El segundo realiza la estimación del modelo estructural, donde se evalúan las relaciones entre los constructos, a través de los coeficientes path y su nivel de significancia. Sin embargo, dado que el objetivo de este trabajo es validar el instrumento propuesto, solamente se analiza el modelo de medida, el cual permite evaluar la validez de dicho cuestionario.

En contraste, el PLS-SEM -también llamado modelo de ruta- es utilizado principalmente para desarrollar teorías en investigación exploratoria. Este lo hace enfocándose en explicar la varianza en las variables dependientes cuando se examina el modelo (Hair et ál., 2016). Para esta investigación la parte que tiene que ver con la modelación de las variables a través de PLS solo es una propuesta para dar continuidad a la investigación sobre el tema de la RSE, ya que en esta investigación la variable se considera de segundo orden.

\section{Fiabilidad}

La determinación de la fiabilidad se realizó a través del alfa de Cronbach, apoyados en la herramienta estadística Smart-PLS, versión 3, el objetivo es verificar que la mayor parte de los ítems diseñados para cada variable independiente permitan alcanzar alfas con valores aceptables, es decir superiores a 0,70 . 
TABLA 8

Cargas factoriales, alfa de Cronbach, fiabilidad compuesta y varianza

\begin{tabular}{|c|c|c|c|c|}
\hline Variable & $\begin{array}{c}\text { Carfas } \\
\text { factoriales }\end{array}$ & $\begin{array}{c}\text { Alfa de } \\
\text { Cronbach }\end{array}$ & $\begin{array}{l}\text { Fiabilidad } \\
\text { compuesta }\end{array}$ & $\begin{array}{c}\text { Varianza } \\
\text { Extraida Media } \\
\text { (AVE) }\end{array}$ \\
\hline \multirow{6}{*}{ Económica } & E1 0,836 & \multirow{6}{*}{0,889} & \multirow{6}{*}{0,916} & \multirow{6}{*}{0,645} \\
\hline & E3 0,726 & & & \\
\hline & E4 0,870 & & & \\
\hline & E5 0,775 & & & \\
\hline & Es 0,768 & & & \\
\hline & E9 0,833 & & & \\
\hline \multirow{4}{*}{ Social } & S11 0,878 & \multirow{4}{*}{0,907} & \multirow{4}{*}{0,931} & \multirow{4}{*}{0,730} \\
\hline & $\mathrm{S} 120,842$ & & & \\
\hline & $\mathrm{S} 130,821$ & & & \\
\hline & $\mathrm{S} 150,846$ & & & \\
\hline \multirow{5}{*}{ Medioambiental } & M21 0,886 & \multirow{5}{*}{0,869} & \multirow{5}{*}{0,910} & \multirow{5}{*}{0,717} \\
\hline & M22 0,908 & & & \\
\hline & $\mathrm{M} 240,183$ & & & \\
\hline & M25 0,837 & & & \\
\hline & M26 0,823 & & & \\
\hline
\end{tabular}

Fuente: elaboración propia.

Como se puede observar en la tabla el Alfa de Cronbach y la fiabilidad compuesta para todas las dimensiones de la RSE (variables), son superiores a 0,750, tienen una alta consistencia interna en las tres dimensiones analizadas. En otras palabras, estos resultados confirman que las dimensiones empleadas para medir las dimensiones económica, social y medioambiental son fiables, lo que verifica por tanto la consistencia interna de dichas dimensiones (variables).

Se considerará que existe validez convergente cuando la Varianza Extraída Media (AVE) alcanza valores superiores a 0,50 (Fornell \& Larcker, 1981; Chin, 1998). Por lo tanto, los resultados estadísticos obtenidos permiten confirmar la existencia de validez convergente en las tres dimensiones analizadas. Al comprar los ítmes propuestos a través del Smart-PLS estos coinciden en los ítems a eliminar del instrumento (instrumento de medición de las dimensiones de RSE), por lo tanto, el instrumento es validado por dos herramientas estadísticas reconocidas por la literatura en el ámbito de las ciencias sociales.

\section{Colinealidad}

Un alto índice de colinealidad puede desestabilizar el modelo y suponer un problema de multicolinealidad, algunos investigadores recomiendan que el valor en constructos o dimensiones formativas sea inferior a 3,5; lo que indicaría la ausencia de multicolinealidad (Petter, Straub \& Rai, 2007), otros trabajos como Diamantopoulos y Siguaw (2006) quienes sugieren que es posible considerar valores de VIF inferiores a 10 para investigaciones empíricas.

TABLA 9

Índice de inflación de la varianza

\begin{tabular}{|c|c|}
\hline Variable & FIV \\
\hline Económica & 1,730 \\
\hline Social & 1,362 \\
\hline Medioambiental & 1,573 \\
\hline
\end{tabular}

Fuente: elaboración propia. 
Para esta investigación el valor más alto de FIV se puede observar en la tabla 9, el resultado fue de 1.730 para la dimensión económica de la RSE, muy inferior al valor 10 fijado por Diamantopoulos y Siguaw (2006), lo que nos lleva a confirmar la ausencia de multicolinealidad para todas las variables del estudio.

\section{Modelo estadístico propuesto}

Finalmente, se realiza una propuesta de modelación a partir de la validación del instrumento para las tres dimensiones de la RSE, para analizar la significación de los pesos de las dimensiones se ha empleado el estadístico $t$ de Student, las tres dimensiones de la RSE muestran una alta contribución en la medición de la variable latente.

TABLA 10

Resultados del modelo

\begin{tabular}{lcc}
\hline Modelo & Beta & R cuadrado \\
\hline Económica & 0,101 & \\
Social & 0,577 & 0,669 \\
Medioambiental & 0,296 & \\
\hline
\end{tabular}

Como se observa en la estructura del modelo propuesto, la regresión PLS es un enfoque basado en regresión que explora las relaciones lineares entre las variables independientes (dimensiones económica, social y medioambiental) y la variable independiente RSE como variable de segundo orden, como ya se habia mencionado anteriormente, el constructo es de tipo reflectivo-formativo.

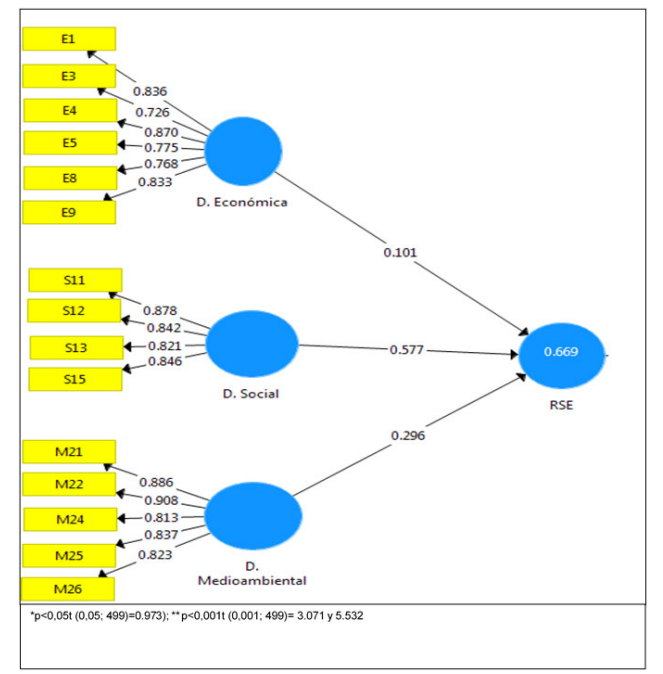

FIGURA 1. Dimensionalidad de RSE

Fuente: elaboración propia.

El valor de $\mathrm{R}^{2}$ es de 0,669 de acuerdo con Granger y Newbold (1976), es posible trabajar con modelos con $\mathrm{R}^{2}$ superiores a 0,25 , aclarando que cuanto mayor sea el valor de la $\mathrm{R}^{2}$ el modelo tendrá mejor ajuste. Las ßetas del modelo señalan que la dimensión social es la que aporta mayor explicación al modelo propuesto con una $\beta=0.577$, de acuerdo Granger y Newbold (1976), es posible explicar modelos con resultados a partir de 0,250 , identificando dicho valor como una explicación debil y betas entre $0,251 \mathrm{y}$ 0,500 aportan una explicación moderada del modelo, valores entre 0,501 y 0,900 aportan una alta explicación.

En cuanto a los resultados de la Beta para la variable independiente de la dimensión social, se observa un resultado por debajo de los datos estadisticamente aceptables, por lo que es importante realizar una nueva revisión en la selección de los ítems para esta variable que permita obtener betas superiores al 0,250. 
Finalmente, para analizar la significación de los pesos de las dimensiones se ha empleado el estadístico $t$ de Student. Tal y como se observa en la figura 1, las dimensión económica de la RSE muestran una baja contribución en la medición de la variable latente.

\section{Conclusiones}

Este artículo presenta un instrumento de medición de la responsabilidad social empresarial (RSE), que fue validado con éxito a través de tres dimensiones de la misma, y con base en ello se confirmaron los ítems para cada constructo. Para este proceso se utilizaron dos herramientas estadisticas reconocidas por las ciencia sociales: SPSS, versión 21 y Smart-PLS, versión 3.

De los resultados se desprenden las conclusiones estadísticas de las cargas factoriales tanto para SPSS como para Smart-PLS, en los cuales se identifican los mismos 15 ítems a considerar dentro del instrumento, el cual se estructuró a través de tres dimensiones. De acuerdo con el resultado estadístico la dimensión económica demuestra una debil aportación a la medida de la precepción de la implementación de la RSE en las empresas con una beta de 0,101 . Por su parte, la dimensión social fue la que quedó comprobada con menos ítems para su estudio, por lo que se pueden tomar dos caminos, ya sea diseñar más ítems para medir esta dimensión social y tener un instrumento de medida equilibrado con el número de ítems por cada dimensión.

De acuerdo con Vicente, Tamayo e Izaguirre (2012), existen factores que puede dificultar la obtención de uniformidad en los resultados los cuales pueden ser el período temporal contemplado en el estudio, la heterogeneidad de las muestras, el tamaño reducido de la muestra, las técnicas empleadas para la medición, el tamaño de la empresa, entre otros.

Los resultados obtenidos hasta el momento son un insumo para dar continuidad a la investigación sobre la RSE que implementa las empresas mexicanas, especialmente las que cotizan en la Bolsa Mexicana de Valores. Cabe señalar que es importante tener un equilibrio en el número de preguntas por cada variable del modelo y revisar los ítems de la variable de la dimensión económica la cual tiene un beta inferior al 0,250 y baja significacia de acuerdo con los valores $t$ de Student, en este caso se pueden seguir eliminando ítems para mejorar el resultado o replantear los ítems existentes a fin de mejorar su comprensión.

Una vez analizados y explicados los datos estadisticos sobre la validez, contenido y colinealidad del instrumento, se concluye que el instrumento es confiable y tiene validez para ser implementado en las empresas socialmente responsables. En cuanto al modelo propuesto através de las tres dimensiones de RSE se demuestran resultados estadisticamente aceptables.

La principal limitación que presenta este trabajo es el levantamiento de datos, el cual se aplicó a una pequeña población y además el bajo número cuestionarios de respuesta obtenido por parte de las empresas, lo que probablemente haya influido en la eliminación de algunos de los ítems del cuestionario inicial.

Finalmente, como futuros trabajos de investigación, es posible aplicar este instrumento de medida en otro grupo de empresas, en ciertos sectores específicos de actividad empresarial o incluso zonas geográficas, para comprobar su adaptación y validez.

\section{Referencias}

Alvarado, A,. \& Schlesinger, M. (2008). Dimensionalidad de la Responsabilidad Social Empresarial percibida y sus efectos sobre la imagen y la reputación: una aproximación desde el modelo de Carroll. Estudios Gerenciales, 24(108), 37-60. DOI: https://doi.org/10.1016/S0123-5923(08)70043-3

Bansal, P. (2005). Evolving sustainably: A longitudinal study of corporate sustainable development. Strategic Management Journal, 26(3), 197-218. DOI: https://doi.org/10.1002/smj.441 
Baumgartner, R., \& Ebner, D. (2010). Corporate sustainability strategies: Sustainability profiles and maturity levels. Sustainable Development, 18, 76-89. DOI: https://doi.org/10.1002/sd.447

Bisquerra, R. (1989). Métodos de investigación educativa. Guia práctica, No. 370.7 B57.

Bowen, H. (1953). Social responsibilities of the businessman, No. 3. Harper.

Cajiga, J. (2009). El concepto de responsabilidad social empresarial. Centro Mexicano para la Filantropía. Recuperado el 02 de enero de 2018 de http://www.cemefi.org/esr

Canessa, I., \& García, E. (2005). El ABC de la responsabilidad Social Empresarial en el Perú y en el Mundo. Lima: Editorial Siklos.

Carroll, A. (1979). A three-dimensional conceptual model of corporate performance. Academy of Management Review, 4(4), 497-505. DOI: $10.2307 / 257850$

Cervantes, V. (2005). Interpretaciones del coeficiente de alpha de Cronbach. Avances en Medición, 3, 9-25. http://ww w.humanas.unal.edu.co/psicometria/files/1113/8574/8604/Articulo_1_Alfa_de_Cronbach_9-28_2.pdf

Chin, W. (1998). The partial least squares approach to structural equation modeling. In G. A. Marcoulides (ed.), Modern methods for business research (pp. 295-336). Mahwah, NJ: Lawrence Erlbaum Associates.

Chow, W. \& Chen, Y. (2011). Corporate Sustainable Development: Testing a New Scale Based on the Mainland Chinese Context. Journal of Business Ethics, 105, 519-533. DOI: 10.1007/s10551-011-0983-x

Cronbach, L. (1951). Coefficient alpha and the internal structure of tests. Psychometrika, 16(3), 297-334. DOI: htt ps://doi.org/10.1007/BF02310555

De la Cuesta, M. (2004). El porqué de la responsabilidad social corporativa. Boletín Económico del ICE, $\mathrm{N}^{\circ} 2813$ 45-58. DOI: nd.

Deephouse, D. (1996). Does isomorphism legitimate? Academy of Management Journal, 39(4), 1024-1039. DOI: h ttps://doi.org/10.5465/256722

Diamantopoulos, A. \& Siguaw, J. (2006). Formative versus reflective indicators in organizational measure development: A comparison and empirical ilustration. British Journal of Management, 17(4), 263-282. DOI: $\mathrm{h}$ ttps://doi.org/10.1111/j.1467-8551.2006.00500.x

Donate, M., \& Guadamillas, F. (2011). Organizational factors to support knowledge management and innovation. Journal of Knowledge Management, 15(6), 890-914. DOI: https://doi.org/10.1108/13673271111179271.

Fornell, C., \& Larcker, D. (1981). Structural equation models with unobservable variables and measurement error: Algebra and statistics. Journal of Marketing Research, 18, 382-388. DOI: http://dx.doi.org/10.2307/3150980

Gallardo-Vázquez, D., Sánchez-Hernández, M., \& Corchuelo-Martínez-Azua, M. (2013). Validación de un instrumento de medida para la relación entre la orientación a la Responsabilidad Social Corporativa y otras variables estratégicas de la empresa. Revista de Contabilidad, 16(1), 11-23. DOI: https://doi.org/10.1016/S11 38-4891(13)70002-5

García de los Salmones, M., Herrero, A., \& Rodríguez, I. (2005). Influence of Corporate Social Responsibility on Loyalty and Valuation of Services. Journal of Business Ethics, 61, 369-385. DOI: 10.1007/s10551-005-5841-2

García-Jiménez, J., Ruiz-de-Maya, S., \& López-López, I. (2017). The impact of congruence between the CSR activity and the company's core business on consumer response to CSR. Spanish Journal of Marketing-ESIC, 21, 26-38. DOI: https://doi.org/10.1016/j.sjme.2017.01.001

Gefen, D., \& Straub, D. (2005). A practical guide to factorial validity using PLS-graph: Tutorial and annotated example. Communications of the Association for Information Systems, 16, 91-109. DOI: 10.17705/1CAIS.01605

Granger, C., \& Newbold, P. (1976). R2 and the Transformation of the regression variables. Journal of Econometrics, Vol(4), (3) 205-210. DOI: https://doi.org/10.1016/0304-4076(76)90032-4

Hair Jr, J., Hult, G., Ringle, C., \& Sarstedt, M. (2016). A primer on partial least squares structural equation modeling (PLS-SEM). Thousand Oaks, CA: Sage Publications.

Hull, C., \& Rothenberg, S. (2008). Firm performance: The interactions of corporate social performance with innovation and industry differentiation. Strategic Management Journal, 29, 781-789. DOI: https://doi.org/10 $.1002 / \mathrm{smj} .675$ 
Jackson, G., \& Apostolakou, A. (2010). Corporate social responsibility in Western Europe: An institutional mirror or substitute? Journal of Business Ethics, 94(3), 371-394. DOI: https://doi.org/10.1007/s10551-009-0269-8

Jarvis, C., MacKenzie, S., \& Podsakoff, P. (2003). A critical review of construct indicators and measurement model misspecification in marketing and consumer research. Journal of Consumer Research, 30(2), 199-218. DOI: htt p://dx.doi.org/10.1086/376806

Kurapatskie, B., \& Darnall, N. (2013). Which corporate sustainability activities are associated with greater financial payoffs? Business strategy and the environment, 22(1), 49-61. DOI: https://doi.org/10.1002/bse.1735

Larrán, M., Herrera, J., \& Martínez, D. (2013). Relación entre la RSE y el performance competitive en la pequeña y mediana empresa: Un estudio empírico. AECA Revista de la Asociación Española de Contabilidad y Administración de Empresas, 104, 9-12. DOI: 10.5295/cdg.120360jh

Marín, R., \& Rubio, B. (2008). La responsabilidad social corporativa como determinante del éxito competitivo: un análisis empírico. Revista Europea de Dirección y Economia de la Empresa, 17(3), 27-42. https://dialnet.unirioj a.es/servlet/articulo?codigo $=2725440$

Mishra, S., \& Suar, D. (2010). Does corporate social responsibility influence firm performance of Indian companies? Journal of Business Ethics, 95(4), 571-601. DOI: https://doi.org/10.1007/s10551-010-0441-1

Orlitzky, M., Schmidt, F. \& Rynes, S. (2003). Corporate social and financial performance: a meta-analysis. Organization Studies, 24(3), 403-411. DOI: https://doi.org/10.1177/0170840603024003910

Panwar, R., Rinne, T., Hansen, E., \& Juslin, H. (2006). Corporate responsibility: Balancing economic, environmental, and social issues in the forest products industry. Forest Products Journal, 56(2), 4-12. DOI: nd.

Petter, S., Straub, D., \& Rai, A. (2007). Specifying formative constructs in information systems research. MIS Quarterly, 31(4), 623-656. DOI: 10.2307/25148814

Porter, M., \& Kramer, M. (2006). Estrategia y sociedad: el vínculo entre ventaja competitiva y la responsabilidad social corporativa. Harvard Business Review, América Latina. Recuperado de http://www.slideshare.net/velugarcete/ RSE-estrategiaysociedadporterykramer

Ramos, M., Manzanares, M., \& Gómez, F. (2014). El efecto del papel mediador de la reputación corporativa en la relación entre la RSC y los resultados económicos. Revista de Estudios Empresariales. Segunda Época, 1, 67-89. https://revistaselectronicas.ujaen.es/index.php/REE/article/view/1378/1200

Rhou, Y., Singal, M., \& Koh, Y. (2016). CSR and financial performance: The role of CSR awareness in the restaurant industry. International Journal of Hospitality Management, 57, 30-39. DOI: 10.1016/j.ijhm.2016.05.007

Rodrigues, M., \& Mendes, L. (2018). Mapping of the literature on social responsibility in the mining industry: A systematic literature review. Journal of Cleaner Production, 181, 88-101. DOI: http://dx.doi.org/10.1016/j.jcl epro.2018.01.163

Saeidi, S., Sofian, S., Saeidi, P., Saeidi, S., \& Saeidi, S. (2015). How does corporate social responsibility contribute to firm financial performance? The mediating role of competitive advantage, reputation, and customer satisfaction. Journal of Business Research, 68(2), 341-350. DOI: 10.1016/j.jbusres.2014.06.024

Sánchez, R., \& Gómez, C. (1998). Conceptos básicos sobre validación de escalas. Revista Colombiana de Psiquiatría, 27, 121-130. http://psiquiatria.org.co/web/wp-content/uploads/2012/04/VOL-27/2/Conceptos\%20b\%C3 \%A1sicos\%20sobre\%20validaci\%C3\%B3n\%20de\%20escalas.pdf

Spiller, R. (2000). Ethical business and investment: A model for business and society.Journal of Business Ethics, 27(1-2), 149-160. DOI: https://doi.org/10.1023/A:1006445915026

Surroca, J., Tribó, J., \& Waddock, S. (2010). Corporate responsibility and financial performance: The role of intangible resources. Strategic Management Journal, 31(5), 463-490. DOI: https://doi.org/10.1002/smj.820

Trump, C., \& Guenther, T. (2017). Too Little or too much? Exploring U-shaped relationships between corporate environmental performance and corporate financial performance. Business Strategy and the Environment, 26(1), 49-68. DOI: https://doi.org/10.1002/bse.1900

Tsoutsoura, M. (2004). Corporate Social Responsibility and Financial Performance. Applied Financial Project Haas School of Business. Berkel: University of California. 
Vázquez, D., \& Hernández, M. (2013). Análisis de la incidencia de la Responsabilidad Social Empresarial en el éxito competitivo de las microempresas y el papel de la innovación. Universia Business Review, 38, 14-31. DOI: nd.

Vicente, M., Tamayo, U., \& Izagirre, J. (2012). Revisión de la metodología empleada y resultados alcanzados en la investigación sobre actuación medioambiental de la empresa y rendimiento económico, 1972-2009. Revista de Métodos Cuantitativos para la Economia y la Empresa, 14, 5-35. DOI: http://dx.doi.org/10.18601/01233734 .n27.08

Vives, A. (2010). La responsabilidad social de las empresas: enfoques ante la crisis. Madrid: Fundación Carolina.

Xie, X., Jia, Y., Meng, X., \& Li, C. (2017). Corporate social responsibility, customer satisfaction, and financial performance: The moderating effect of the institutional environment in two transition economies. Journal of Cleaner Production, 150, 26-39. DOI: 10.1016/j.jclepro.2017.02.192

\section{Notas}

* Artículo de investigación científica y tecnológica.

\section{Licencia Creative Commons CC BY 4.0}

Para citar este artículo: Landázuri A., Y., Hinojosa C., A., \& Aguilar M., N. (2018). Responsabilidad Social Empresarial: un instrumento para medir la implementación en las empresas del índice de sustentabilidad de la Bolsa Mexicana de Valores. Cuadernos de Contabilidad, 19(48), 1-16. https://doi.org/10.11144/Javeri ana.cc19-48.rsei 